\title{
GENERALIZED UNCERTAINTY PRINCIPLE AND DARK MATTER
}

\author{
Pisin Chen \\ Stanford Linear Accelerator Center \\ Stanford University, Stanford, CA 94309, USA
}

\begin{abstract}
There have been proposals that primordial black hole remnants (BHRs) are the dark matter, but the idea is somewhat vague. Recently we argued that the generalized uncertainty principle (GUP) may prevent black holes from evaporating completely, in a similar way that the standard uncertainty principle prevents the hydrogen atom from collapsing. We further noted that the hybrid inflation model provides a plausible mechanism for production of large numbers of small black holes. Combining these we suggested that the dark matter might be composed of Planck-size BHRs. In this paper we briefly review these arguments, and discuss the reheating temperature as a result of black hole evaporation.
\end{abstract}

\section{INTRODUCTION}

It is by now widely accepted that dark matter $(\mathrm{DM})$ constitutes a substantial fraction of the present critical energy density in the universe. However, the nature of DM remains an open problem. There exist many DM candidates, among which a contending category is weakly interacting massive particles, or WIMPs. It has been suggested that primordial black holes (PBHs) [1,2] are a natural candidate for WIMPs [3]. More recent studies [4] based on the PBH production from the "blue spectrum" of inflation demand that the spectral index $n \sim 1.3$, but this possibility may be ruled out by the recent WMAP experiment [5].

In the standard view of black hole thermodynamics, based on the entropy expression of Bekenstein [6] and the temperature expression of Hawking [7], a small black hole should emit blackbody radiation, thereby becoming lighter and hotter, leading to an explosive end when the mass approaches zero. However Hawking's calculation assumes a classical background metric and ignores the radiation reaction, assumptions which must break down as the black hole becomes very small and light. Thus it does not provide an answer as to whether a small black hole should evaporate entirely, or leave something else behind, which we refer to as a black hole remnant (BHR).

Numerous calculations of black hole radiation properties have been made from different points of view [8], and some hint at the existence of remnants, but none appears to give a definitive answer. A cogent argument against the existence of BHRs can be made [9]: since there is no evident symmetry or quantum number preventing it, a black hole should radiate entirely away to photons and other ordinary stable particles and vacuum, just like any unstable quantum system.

In a series of recent papers [10,11], a generalized uncertainty principle (GUP) [12-14] was invoked to argue the contrary, that the total collapse of a black hole may be prevented by dynamics and not by symmetry, just like the prevention of hydrogen atom from collapse by the uncertainty principle [15]. These arguments then lead to a modified black hole entropy and temperature, and as a consequence the existence of a BHR at around the Planck mass. This notion was then combined with hybrid inflation model [16-19] and it was shown that primordial BHRs might in principle be the primary source for dark matte [11]. In this paper we briefly reproduce these arguments, and include additional discussion on the reheating temperature as a result of black hole evaporation.

\section{GENERALIZED UNCERTAINTY PRINCIPLE}

As a result of string theory [12] or general considerations of quantum mechanics and gravity [13,14], the GUP gives the position uncertainty as

$$
\Delta x \geq \frac{\hbar}{\Delta p}+l_{p}^{2} \frac{\Delta p}{\hbar}
$$

where $l_{p}=\left(G \hbar / c^{3}\right)^{1 / 2} \approx 1.6 \times 10^{-33} \mathrm{~cm}$ is the Planck length. A heuristic derivation may also be made on dimensional grounds. We think of a particle such as an electron being observed by means of a photon with momentum $p$. The usual Heisenberg argument leads to an electron position uncertainty given by the first term in Eq.(1). But we should add to this a term due to the gravitational interaction of the electron with the photon, and that term must be proportional to $G$ times the photon energy, or $G p c$. Since the electron momentum uncertainty $\Delta p$ will be of order of $p$, we see that 
on dimensional grounds the extra term must be of order $G \Delta p / c^{3}$, as given in Eq.(1). Note that there is no $\hbar$ in the extra term when expressed in this way. The position uncertainty has a minimum value of $\Delta x=2 l_{p}$, so the Planck distance, $l_{p}$, plays the role of a fundamental length.

\section{BLACK HOLE REMNANT}

The characteristic energy $E$ of the emitted photons may be estimated from the uncertainty principle. In the vicinity of the black hole surface there is an intrinsic uncertainty in the position of any particle of about the Schwarzschild radius, $\Delta x \approx r_{s}$, due to the behavior of its field lines [20] - as well as on dimensional grounds. This leads to a momentum uncertainty

$$
\Delta p \approx \frac{\hbar}{\Delta x}=\frac{\hbar}{r_{s}}=\frac{\hbar c^{2}}{2 G M_{\mathrm{BH}}},
$$

and hence to an energy uncertainty of $\Delta p c \approx \hbar c^{3} / 2 G M_{\mathrm{BH}}$. We identify this as the characteristic energy of the emitted photon, and thus as a characteristic temperature; it agrees with the Hawking temperature up to a factor $4 \pi$, which we will henceforth include as a "calibration factor" and write (with $k_{B}=1$ ),

$$
T_{\mathrm{H}} \approx \frac{\hbar c^{3}}{8 \pi G M_{\mathrm{BH}}}=\frac{M_{p}^{2} c^{2}}{8 \pi M_{\mathrm{BH}}},
$$

where $M_{p}=(\hbar c / G)^{1 / 2} \approx 1.2 \times 10^{19} \mathrm{GeV}$ is the Planck mass.

The blackbody energy output rate of $\mathrm{BH}$ is given by

$$
\dot{x}=\frac{1}{t_{c h}\left(x_{i}^{3}-3 t / t_{c h}\right)^{2 / 3}},
$$

where $x=M_{\mathrm{BH}} / M_{p}$ and $x_{i}$ refers to the initial mass of the hole. $t_{c h}=60(16)^{2} \pi t_{p} \approx 4.8 \times 10^{4} t_{p}$ is a characteristic time for $\mathrm{BH}$ evaporation, and $t_{p}=\left(\hbar G / c^{5}\right)^{1 / 2} \approx 0.54 \times 10^{-43}$ sec is the Planck time. The black hole thus evaporates to zero mass in a time given by $t / t_{c h}=x_{i}^{3} / 3$, and the rate of radiation has an infinite spike at the end of the process.

The momentum uncertainty according to the GUP is

$$
\frac{\Delta p}{\hbar} \approx \frac{\Delta x}{2 l_{p}^{2}}\left[1 \mp \sqrt{1-4 l_{p}^{2} /(\Delta x)^{2}}\right] .
$$

Therefore the modified black hole temperature becomes

$$
T_{\mathrm{GUP}}=\frac{M_{p} c^{2}}{4 \pi} x\left[1 \mp \sqrt{1-1 / x^{2}}\right] .
$$

This agrees with the Hawking result for large mass if the negative sign is chosen, whereas the positive sign has no evident physical meaning. Note that the temperature becomes complex and unphysical for mass less than the Planck mass and Schwarzschild radius less than $2 l_{p}$. At the Planck mass the slope is infinite, which corresponds to zero heat capacity of the black hole, and the evaporation comes to a stop.

If there are $g$ species of relativistic particles, then the $\mathrm{BH}$ evaporation rate is

$$
\dot{x}=-\frac{16 g}{t_{c h}} x^{6}\left[1-\sqrt{1-1 / x^{2}}\right]^{4} .
$$

Thus the hole with an initial mass $x_{i}$ evaporates to a Planck mass remnant in a time given by

$$
\begin{aligned}
\tau & =\frac{t_{c h}}{16 g}\left[\frac{8}{3} x_{i}^{3}-8 x_{i}-\frac{1}{x_{i}}+\frac{8}{3}\left(x_{i}^{2}-1\right)^{3 / 2}-4 \sqrt{x_{i}^{2}-1}+4 \cos ^{-1} \frac{1}{x_{i}}+\frac{19}{3}\right] \\
& \approx \frac{x_{i}^{3}}{3 g} t_{c h}, \quad x_{i} \gg 1
\end{aligned}
$$

The energy output given by Eq.(7) is finite at the end point where $x=1$, i.e., $d x /\left.d t\right|_{x=1}=-16 g / t_{c h}$, whereas for the Hawking case it is infinite at the endpoint where $x=0$. The present result thus appears to be more physically reasonable. The evaporation time in the $x_{i} \gg 1$ limit agrees with the standard Hawking picture. 


\section{HYBRID INFLATION AND BLACK HOLE PRODUCTION}

The hybrid inflation, first proposed by A. Linde [16], can naturally induce large number of small PBHs [21]. In the hybrid inflation model two inflaton fields, $(\phi, \psi)$, are invoked. Governed by the inflation potential, $\phi$ first executes a "slow-roll" down the potential, and is responsible for the more than 60 e-folds expansion while $\psi$ remains zero. When $\phi$ eventually reduces to a critical value, it triggers a phase transition that results in a "rapid-fall" of the energy density of the $\psi$ field, which lasts only for a few e-folds, that ends the inflation.

The evolution of the $\psi$ field during the second stage inflation, measured backward from the end, is

$$
\psi(N[t])=\psi_{e} \exp (-s N[t])
$$

where $N(t)=H_{*}\left(t_{e}-t\right)$ is the number of e-folds from $t$ to $t_{e}, H_{*}$ is the Hubble parameter during inflation, and $s$ is a numerical factor of the order unity.

Quantum fluctuations of $\psi$ induce variations of the starting time of the second stage inflation, i.e., $\delta t=\delta \psi / \dot{\psi}$. This translates into perturbations on the number of e-folds, $\delta N=H_{*} \delta \psi / \dot{\psi}$, and therefore the curvature contrasts, $\delta \rho / \rho \equiv \delta$. With an initial density contrast $\delta(m) \equiv \delta \rho /\left.\rho\right|_{m}$, the probability that a region of mass $m$ becomes a PBH is $[23]$

$$
P(m) \sim \delta(m) e^{-w^{2} / 2 \delta^{2}}
$$

Let us assume that the universe had inflated $e^{N_{c}}$ times during the second stage of inflation. It can be shown [21] that

$$
e^{N_{c}} \sim\left(\frac{2 M_{p}}{s H_{*}}\right)^{1 / s}
$$

and the curvature perturbations reentered the horizon at time

$$
t \sim t_{h}=H_{*}^{-1} e^{3 N_{c}}
$$

At this time if the density contrast was $\delta \sim 1$, then BHs with size $r_{s} \sim H_{*}^{-1} e^{3 N_{c}}$ would form with an initial mass

$$
M_{\mathrm{BH} i} \simeq \frac{M_{p}^{2}}{H_{*}} e^{3 N_{c}} .
$$

Following the numerical example given in Ref.21, we let $H_{*} \sim 5 \times 10^{13} \mathrm{GeV}$ and $s \sim 3$. Then the density contrast can be shown to be $\delta \sim 1 / 7$, and the fraction of matter in the $\mathrm{BH}$ is thus $P(m) \sim 10^{-2}$. From Eq. (11), $e^{N_{c}} \sim 54$. So the total number of e-folds is $N_{c} \sim 4$. The black holes were produced at the moment $t_{h} \sim 2 \times 10^{-33}$ sec, and had a typical mass $M_{\mathrm{BH} i} \sim 4 \times 10^{10} M_{p}$. Let $g \sim 100$. Then the time it took for the BHs to reduce to remnants, according to Eq. (8), is

$$
\tau \sim \frac{x_{i}^{3}}{3 g} t_{c h} \sim 5 \times 10^{-10} \mathrm{sec} .
$$

The "black hole epoch" thus ended in time for baryogenesis and other subsequent epochs in the standard cosmology. As suggested in Ref.21, such a post-inflation PBH evaporation provides an interesting mechanism for reheating.

\section{BLACK HOLE REMNANTS AS DARK MATTER}

This process also provides a natural way to create cold dark matter. Although in our example $P(m) \sim 10^{-2}, \mathrm{PBHs}^{-}$ would soon dominate the energy density by the time $t \sim P(m)^{-2} t_{h} \sim 2 \times 10^{-29}$ s, because the original relativistic particles would be diluted much faster than non-relativistic PBHs. By the time $t \sim \tau$, all the initial BH mass $\left(x_{i}\right)$ had turned into radiation except one unit of $M_{p}$ preserved by each BHR. As BH evaporation rate rises sharply towards the end, the universe at $t \sim \tau$ was dominated by the $\mathrm{BH}$ evaporated radiation.

Roughly, $\Omega_{\mathrm{BHR}, \tau} \sim 1 / x_{i}$ and $\Omega_{\gamma, \tau} \sim 1$ at $t \sim \tau$, and since the universe resumed its standard evolution after the black hole epoch $(t>\tau)$, we find the density parameter for the BHR at present to be 


$$
\Omega_{\mathrm{BHR}, 0} \sim\left(\frac{t_{e q}}{\tau}\right)^{1 / 2}\left(\frac{t_{0}}{t_{e q}}\right)^{2 / 3} \frac{1}{x_{i}} \Omega_{\gamma, 0}
$$

where $t_{0} \sim 4 \times 10^{17} \mathrm{~s}$ is the present time, and $t_{e q}$ is the time when the density contributions from radiation and matter were equal. It is clear from our construction that $\left(t_{e q} / \tau\right)^{1 / 2} \sim x_{i}$. So $t_{e q} \sim 10^{12}$ sec, which is close to what the standard cosmology assumes, and Eq.(15) is reduced to a simple and interesting relationship:

$$
\Omega_{\mathrm{BHR}, 0} \sim\left(\frac{t_{0}}{t_{e q}}\right)^{2 / 3} \Omega_{\gamma, 0} \sim 10^{4} \Omega_{\gamma, 0} .
$$

In the present epoch, $\Omega_{\gamma, 0} \sim 10^{-4}$. So we find $\Omega_{\mathrm{BHR}, 0} \sim \mathcal{O}(1)$, about the right amount for dark matter!

\section{BLACK HOLE EPOCH AND REHEATING TEMPERATURE}

As discussed above, shortly after PBHs were produced the density of the universe was dominated by the BHs. Eventually the universe was reheated through their continuous evaporation. To simplify the discussion we ignore BH accretions of the radiation as well as $\mathrm{BH}$ mergers. Then under Hubble expansion the effective reheating temperature at the end of the black hole epoch, or $t \sim \tau$, can be expressed as

$$
T_{r}\left(\tau\left[x_{i}\right]\right)=\frac{1}{x_{i}-1} \int_{1}^{x_{i}} d x T_{\mathrm{GUP}}(x) \frac{a(t[x])}{a\left(\tau\left[x_{i}\right]\right)},
$$

where $a(t)$ is the scale factor. Since $x_{i} \gg 1$, the evaporation only became effective near the late times during this black hole epoch, when the energy density was dominated by the BH radiation. As a further approximation we assume radiation dominance throughout the $\mathrm{BH}$ epoch so that $a(t) \propto t^{1 / 2}$. Expressing $t$ in terms of $x$ using Eq.(7), we find

$$
T_{r}\left(\tau\left[x_{i}\right]\right) \approx \frac{M_{p} c^{2}}{16 \pi\left(x_{i}-1\right)}\left[2 \log \left(2 x_{i}\right)-1\right]+\mathcal{O}\left(\frac{1}{x_{i}^{3}}\right) .
$$

In our model $x_{i} \sim 4 \times 10^{10}$. So $T_{r}\left(\tau\left[x_{i}\right]\right) \sim 1.3 \times 10^{8} \mathrm{GeV}$, which is sufficiently lower than the Planck and the GUT scales, but higher than the baryogenesis scale.

\section{ACKNOWLEDGEMENTS}

I deeply appreciate my early collaborations and fruitful discussions with Ronald J. Adler. I also thank S. Dimopoulos, A. Green, A. Linde, M. Shmakova, and K. Thompson for helpful discussions. This work is supported by the Department of Energy under Contract No. DE-AC03-76SF00515.

[1] Ya. B. Zeldovich and I. D. Novikov, Sov. Astron. 10, 602 (1966).

[2] S. W. Hawking, Mon. Not. R. Astron. Soc. 152, 75 (1971).

[3] J. H. MacGibbon, Nature 329, 308 (1987); J. D. Barrow, E. J. Copeland, A. R. Liddle, Phys. Rev. D 46, 645 (1992).

[4] B. J. Carr, J. H. Gilbert, and J. E. Lidsey, Phys. Rev. D 50, 4853 (1994); J. E. Lidsey, B. J. Carr, and J. H. Gilbert, arXiv: astro-ph/9406028.

[5] D. N. Spergel et al., arXiv: astrop-ph/0302209, submitted to Astrophys. J.

[6] J. D. Bekenstein, Phys. Rev. D 7, 2333 (1973); Lett. al Nuovo Cimento 4, 737 (1972).

[7] S. W. Hawking, Comm. Math. Phys. 43, 199 (1974).

[8] See, for example, M. K. Parikh and F. Wilczek, Phys. Rev. Lett. 85, 5042 (2000), and references therein.

[9] L. Susskind, private communications (2001); J. Math. Phys. 36, 6377 (1995).

[10] R. J. Adler, P. Chen, and D. Santiago, Gen. Rel. Grav. 33 (12), 2101 (2001).

[11] P. Chen, arXiv: astro-ph/0303349; submitted to Phys. Rev. Lett.

[12] G. Veneziano, Europhys. Lett. 2, 199 (1986); E. Witten, Phys. Today, Apr. 24 (1996).

[13] R. J. Adler and D. I. Santiago, Mod. Phys. Lett. A14, 1371 (1999). 
[14] M. Maggiore, Phys. Lett. B304, 65 (1993); F. Scardigli, Phys. Lett. B452, 39 (1999).

[15] R. Shankar, Principles of Quantum Mechanics, 2nd ed. (Plenum, 1994).

[16] A. Linde, Phys. Lett. B259, 38 (1991); Phys. Rev. D 49, 748 (1994).

[17] E. J. Copeland, A. R. Liddle, D. H. Lyth, E. D. Stewart, and D. Wands, Phys. Rev. D 49, 6410 (1994).

[18] L. Randall, M. Soljacic, and A. H. Guth, Nucl. Phys. 472, 408 (1996).

[19] For a recent review, see D. H. Lyth and A. Riotto, Phys. Rep. 314, 1 (1999); A. Linde, Phys. Rep. 333-334, 575 (2000).

[20] R. S. Hanni and R. Ruffini, in Black Holes, eds. C. DeWitt and B. S. DeWitt, R57 (Gordon and Breach, 1973); R. J. Adler and T. K. Das, Phys. Rev. D 14, 2474 (1976).

[21] J. Garcia-Bellido, A. Linde, and D. Wands, Phys. Rev. D 54, 6040 (1996).

[22] A. R. Liddle and D. H. Lyth, Phys. Rep. 231, 1 (1993).

[23] B. J. Carr and S. W. Hawking, Mon. Not. R. Astron. Soc. 168, 399 (1974); B. J. Carr, Astrophys. J. 201, 1 (1975). 